\title{
International
}

\section{International Alumni Networking Grant Awards}

\begin{abstract}
Tn February 2014, APSA awarded professional development grants to three _Africa workshop alumni, totaling approximately \$3,0oo. Recipients included Fatai Aremu, '12, Edmond Mballa Elanga, '13, and Henry Kam Kah,'o9. To date, APSA has awarded more than $\$ 40,000$ in Alumni Networking Grants.
\end{abstract}

APSA's Alumni Networking Grants are available primarily for Africa Workshops alumni. With support from the Andrew W. Mellon Foundation, $\$ 45,000$ is available each year to support alumni participation in professional development opportunities, facilitate networking potential, and encourage collaboration among scholars and academic institutions across Africa. Applications will be accepted on a rotating basis for three types of grants: professional development grants, workshop grants, and publication grants.

Application and program information can be found on the Africa Workshop website at http://community.apsanet.org/Africa/alumnigrants. Questions should be directed to SiadatIlo at africaworkshops@apsanet.org.

\section{International Membership}

s of April 2014, APSA international membership totaled 2,173. Mutiple countries
with the same number of members are grouped together Membership per country
are noted as follows:
Canada

United Kingdom

Germany

Japan

Australia

Mexico

Switzerland

Netherlands

Israel

France

Italy, Sweden, Korea (Rep)

Brazil

Turkey

Taiwan

Belgium

Norway, Spain

Hong Kong, China

China, Nigeria, Singapore

Ireland

Denmark

Austria, Chile, Hungary,

Russia

Argentina

India, New Zealand

South Africa
373

.326

.196

.168

.. 68

. .55

...53

51

47

... 46

44

40

36

32

29

.27

26

25

21

19

16

15

14

13
Finland ……………………………............... 10

Ghana ......................................................... 9

Colombia, Czech Republic,Greece, Poland, Romania ..............................................

Portugal ...............................................................7

Cameroon, Saudi Arabia, Scotland, Thailand.

Bangladesh, Botswana, Egypt, Kasakhstan, Kenya, Slovenia. Tanzania ..........................5 Cyprus, Ethiopia, Malaysia, Qatar ..............4 Bulgaria, Ecuador, Luxembourg, Macao, Peru, Philippines, Sudan, Uruguay .......3 Albania, Benin, Burkina Faso, Dominion Republic, Estonia, Iraq, Indonesia, Kosovo, Kuwait, Malawi, Morocco, Pakistan, Slovakia,Ukraine, Uganda, West Africa, Zimbabwe

Armenia, Bahamas, Bahrain, Bolivia, Brunei Darussalem, Burundi, Congo, Djibouti, Fiji, Gabon, Georgia, Greenland, Jamaica Kyrgyzstan, Latvia, Lithuania, Madagascar, Mali, Mauritius, Montenegro, Niger, Panama, Serbia, Sri Lanka, Trinidad \& Tabago, United Arab Emirates, Venezuela, Zambia . 